Original

\title{
Estudio prospectivo no randomizado comparando los resultados funcionales y oncológicos entre la prostatectomía radical retropúbica y laparoscópica
}

\author{
Marcelo Kerkebe Lama*,**, Nelson R. Orellana Salinas*, José M. Flores Martínez**, \\ Rubén A. Olivares Gribbell*, Oscar Storme Cabrera**, Cynthia A. Fuentealba Sudy* \\ *Servicio de Urología del Hospital DIPRECA. **Departamento Urología Universidad de Chile.
}

\begin{abstract}
Resumen
Introducción: La prostatectomía radical es un procedimiento quirúrgico potencialmente curativo. Objetivo: Evaluar y comparar los resultados quirúrgicos y oncológicos entre la prostatectomía radical retropúbica (PRR) y la prostatectomía radical laparoscópica (PRL) en el servicio de Urología del Hospital DIPRECA.

Material y método: Un total de 115 pacientes, 56 para PRL y 59 PRR realizadas entre enero 2003 hasta marzo 2007. Estudio prospectivo, no randomizado. Se compararon los resultados funcionales y oncológicos de acuerdo a variables estandarizadas y su análisis estadístico correspondiente, usando programa estadístico SPSS 12.0.

Resultados: El tiempo operatorio promedio fue de 202,5 minutos para PRL y 150,5 minutos en PRR (p<0,0001). Requerimiento de transfusiones mayor para la PRR $(\mathrm{p}<0,0001)$, al igual que en número de días de hospitalización ( $\mathrm{p}=0,0073$ ) y tiempo de requerimiento de sonda Foley ( $\mathrm{p}=0,0001)$. Hubo 15 complicaciones en PRL y 8 en PRR ( $\mathrm{p}=0,10)$. No hubo diferencias significativas en la conservación de la función sexual y continencia urinaria postcirugía. En el análisis oncológico el margen quirúrgico positivo no presento diferencias, tampoco en la recidiva bioquímica en el seguimiento.

Conclusión: Nuestros resultados muestran que ambas cirugías tienen resultados similares en términos oncológicos, continencia y función sexual. Existe un claro beneficio a favor de la PRL en cuanto a menor sangrado, y recuperación precoz. Si bien la PRR supera a la PRL en tiempo operatorio, creemos que esta variable depende de la curva de aprendizaje. Según nuestra revisión, éste es el primer trabajo comparativo entre estas dos técnicas que se publica en Chile.
\end{abstract}

Palabras clave: Próstata. Cáncer. Cirugía laparoscópica.

\section{Prospective study and comparative of surgical and oncologic outcome between laparoscopic and retropubical radical prostatectomy}

\section{Abstract}

Introduction: Radical prostatectomy (RP) is a potentially healing surgical procedure.

Objective: We evaluate and compare the surgical and oncologic outcomes between laparoscopic and retropubical radical prostatectomy in the Urology Department in DIPRECA Hospital.

Method: We constructed a nonrandomised, prospective study between january 2003 and march 2007. A total of 115 patients, 56 operated laparoscopically and 59 by retropubical RP. Functional and oncologic results were compared according to standardized variables and their corresponding statistical analysis, for which we used SPSS 12.0 program.

Results: Mean operation time was 202,5 minutes for laparoscopic RP and 150,5 for retropubical RP (p<0.0001). Retropubical RP required more blood transfusions $(\mathrm{p}<0.0001)$, longer hospital stay $(\mathrm{p}=0,0073)$ and longer need for vesical catheter $(\mathrm{p}=0,0001)$ than laparoscopic RP. There were 23 complications, 15 attributable to laparoscopic RP. We found no significant differences in postsurgical sexual function and urinary continence. In respect to the oncologic variables, we found no statistically relevant differences in positive surgical margins nor biochemical relapse during follow up.

Conclusion: We found no significant differences between retropubical and laparoscopic RP in the oncologic and functional variables analyzed. Nevertheless, our experience shows a distinct benefit in favour of the laparoscopic approach in relation to bleeding and recovery rate. Though retropubical RP has a shorter operating time, we believe this variable depends on the learning curve still developing for laparoscopic RP. According to our literary review, this is the first publication in Chile that compares both techniques.

Keywords: Prostate. Cancer. Laparoscopic surgery. 
T a prostatectomía radical es el procedimiento Lquirúrgico potencialmente curativo en el tratamiento de cáncer de próstata órgano confinado ${ }^{1}$. Muchos urólogos usan la aproximación retropúbica por ser más familiar con la anatomía quirúrgica, asî como la preservación de bandeletas neurovasculares descritas por Walsh et al. ${ }^{1-3}$.

El estándar quirúrgico en la prostatectomía radical es lograr un buen control del cáncer, manteniendo la continencia urinaria, la potencia sexual y baja morbilidad. La presentación del cáncer de próstata ha cambiado durante los últimos 15 años, debido al screening con antígeno prostático específico. Gracias a este examen el diagnóstico más frecuente en la actualidad es el estado localizado, logrando el diagnóstico en población más joven, con niveles más bajos de antígeno prostático específico, puntaje Gleason menor o igual a 6 y tacto rectal normal (Estadios T1C) ${ }^{1,2}$.

Hay abundantes estudios que avalan la seguridad y eficacia de la prostatectomía radical retropúbica (PRR) y laparoscópica (PRL), esta última con factibilidad técnica demostrada. En 1997 se describen las aproximaciones laparoscópicas en la prostatectomía radical por Schuessler et al, en $1997^{4,5}$, Resultados preliminares han demostrado su factibilidad y un satisfactorio control del cáncer, siendo una alternativa a la aproximación abierta de la prostatectomía radical, con disminución de la morbilidad postoperatoria, disminución del dolor, requerimientos analgésicos, menor estancia hospitalaria y una reintegración a las actividades de los pacientes en forma más rápida ${ }^{2-5}$.

En nuestro país, la experiencia en prostatectomía radical laparoscópica es inicial, seguido por una generación de urólogos interesados en esta nueva técnica quirúrgica en el tratamiento de cáncer de próstata. No existen estudios nacionales comparativos entre prostatectomía radical retropúbica y laparoscópica, sólo publicaciones de experiencias iniciales en esta última técnica. Este trabajo sería, por lo tanto, el primero en comparar ambas técnicas quirúrgicas en el tratamiento del cáncer de próstata localizado en Chile.

\section{OBJETIVO}

El propósito de este trabajo es presentar la evaluación y comparación de los resultados tanto quirúrgicos como oncológicos entre dos técnicas quirúrgicas: la prostatectomía radical retropúbica y laparoscópica en el manejo del cáncer de próstata localizado en el Servicio de Urología del Hospital DIPRECA.

\section{MATERIALES Y MÉTODO}

Para este estudio se incorporaron un total de 115 pacientes pertenecientes al Servicio de Urología del Hospital DIPRECA, entre enero 2003 hasta marzo 2007, todos portadores de cáncer prostático localizado, a los cuales se les realizó prostatectomía radical retropúbica o laparoscópica. Se hizo un seguimiento de los pacientes en forma prospectiva. La técnica quirúrgica a elección dependía del urólogo tratante. Las PRR fueron realizadas por urólogos con curva de aprendizaje finalizada para este procedimiento. Las PRL, en cambio, fueron realizadas por un urólogo (MK) formado en laparoscopía cuya curva de aprendizaje fue desarrollada con los pacientes de este estudio.

De forma preoperatorio todos presentaban evaluación clínica, tacto rectal, antígeno prostático específico y biopsia prostática transrectal ecodirigida, con confirmación del diagnóstico de cáncer y su score de Gleason correspondiente. Se incluyeron los pacientes sin cirugía prostática previa, próstatas menor de $100 \mathrm{~g}$. Gleason menor de 8 y con datos completos para obtener un adecuado seguimiento, de al menos un año.

Las variables analizadas para evaluar factores quirúrgicos fueron tiempo operatorio, transfusión, complicaciones, alta post-cirugía y tiempo uso sonda Foley, además, de función sexual a 1 año y continencia a los 6 meses y 1, 2 y 3 años. Para resultados oncológicos fueron margen quirúrgico positivo y recidiva bioquímica a los 6 meses, 1,2 y 3 años.

Los datos se ingresaron al programa SPSS 12.0 para su análisis estadístico, se aplicó $\mathrm{T}$ student $\mathrm{y}$ Chi cuadrado, se tomaron como diferencias estadísticamente significativas a los $\mathrm{P}$ value menor 0,05 .

\section{RESULTADOS}

En el análisis de ambos grupos no hubo diferencias estadísticamente significativas, lo cual los hace comparables (Tabla 1).

En cuanto al análisis de ambas técnicas quirúrgicas el tiempo operatorio fue de 202,5 $\pm 52,1 \mathrm{minu}-$ tos para PRL y $150,56 \pm 29,93$ en PRR siendo estadísticamente significativo $(\mathrm{p}<0,0001)$.

En cuanto al requerimiento de transfusiones hay diferencias significativas a favor de la prostatectomía 
laparoscópica con un total de 7 pacientes, 23 pacientes requirieron transfusión en prostatectomía retropúbica ( $<<0,0001)$. En número de días de hospitalización promedio fue de $7,25 \pm 4,7 \mathrm{y}$ $10,7 \pm 9,2$ días $(p=0,0073)$ respectivamente. El tiempo de requerimiento de sonda Foley fue de 8,76 $\pm 3,9$ días en

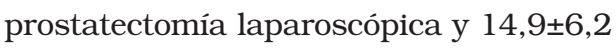
días en retropúbica $(\mathrm{p}=0,0001)$ (Tabla 2).

Se observaron un total de 15 complicaciones en prostatectomía laparoscópica y 8 en retropúbica, de ellas se separaron las derivadas del la técnica quirúrgica 10 y 7 respectivamente; y las secundarias al manejo de la sonda Foley en el post operatorio 5 para la PRL y 1 para PRR, no habiendo diferencias significativas (Tabla 3).

En el análisis de margen quirúrgico positivo tuvimos $28,57 \%$ en prostatectomía laparoscópica y 35,6\% en retropúbica $(\mathrm{p}=0.68)$. La recidiva bioquímica a los 6 meses, 1, 2 y 3 años no fue estadísticamente significativa entre ambos grupos. (Tabla 2).

La incontinencia urinaria a los 6 meses es de 1 paciente en prostatectomía laparoscópica y 2 en prostatectomía retropúbica $(p=0,5997)$, a 1 año de seguimiento no hay casos en prostatectomía laparoscópica y 2 casos en retropúbica $(\mathrm{p}=0,506)$, lo cual se mantiene a los 2 y 3 años, sin diferencias significativas (Tabla 2).

La función sexual se conservó en 15 pacientes y 26 pacientes en prostatectomía laparoscópica y retropúbica respectivamente no habiendo diferencia significativa $(\mathrm{p}=0,0648)$ (Tabla 2$)$.

\section{DISCUSIÓN}

La prostatectomía radical sigue siendo el tratamiento quirúrgico estándar y de elección en el manejo del cáncer de próstata localizado, con un buen control oncológico, baja morbilidad operatoria y postoperatoria traducida en bajos porcentajes de incontinencia urinaria y un aumento en los índices de conservación de la potencia sexual. En recientes años, la prostatectomía radical laparos-
Tabla 1. Distribución por número pacientes, edad, Gleason y estadío clínico del cáncer de próstata localizado entre prostatectomía radical laparoscópica versus retropúbica en el Servicio de Urología del Hospital DIPRECA

\begin{tabular}{lccc}
\hline & $\begin{array}{c}\text { Prostatectomía } \\
\text { laparoscópica }\end{array}$ & $\begin{array}{c}\text { Prostatectomía } \\
\text { retropúbica }\end{array}$ & $\begin{array}{c}\text { Valor } \\
\text { de p }\end{array}$ \\
\hline N & 56 & 59 & NS \\
Edad & 64,4 & 63,5 & NS \\
Gleason Moda (Rango) & $5(3-7)$ & $5(3-7)$ & NS \\
APE & $7,94(1,8-35)$ & $8,85(2,5-34)$ & NS \\
cT1c & 39 & 40 & NS \\
cT2a & 15 & 14 & NS \\
cT2b & 1 & 5 & NS \\
cT2c & 1 & 0 & NS \\
\hline
\end{tabular}

P: P value N: Número Paciente NS: No significativo cT: Estadío Clínico.

Tabla 2. Diferencias entre resultados funcionales y oncológicos entre prostatectomía radical laparoscópica versus retropúbica en el Servicio de Urologia del Hospital DIPRECA

\begin{tabular}{lccc}
\hline & $\begin{array}{c}\text { Prostatectomia } \\
\text { radical } \\
\text { laparoscópica }\end{array}$ & $\begin{array}{c}\text { Prostatectomia } \\
\text { radical } \\
\text { retropúbica }\end{array}$ & $\begin{array}{c}\text { Valor } \\
\text { de p }\end{array}$ \\
\hline Tiempo Operatorio (minutos) & 202,5 & 150,56 & 0,0001 \\
Transfusión (unidad/paciente) & $12 / 7$ & $55 / 23$ & 0,0001 \\
Dias de Hospitalización & 7,25 & 10,72 & 0,0073 \\
Tiempo uso Sonda Foley (días) & 8,76 & 14,9 & 0,0001 \\
Margen quirúrgico positivo & $28,57 \%$ & $35,6 \%$ & 0,6837 \\
Recidiva Bioquimica 6 meses & $10,7 \%$ & $10 \%$ & 0.8995 \\
Recidiva Bioquimica 1 año & $10,7 \%$ & $11,6 \%$ & 0,8709 \\
Recidiva Bioquimica 2 años & $10,7 \%$ & $15 \%$ & 0,9043 \\
Recidiva Bioquimica 3 años & $19,6 \%$ & $20 \%$ & 0,9039 \\
Incontinencia Urinaria 6 meses & 1 pacientes & 2 pacientes & 0,5997 \\
Incontinencia Urinaria 1 año & 0 pacientes & 2 pacientes & 0,5064 \\
Función Eréctil Conservada & 15 pacientes & 26 pacientes & 0,0648 \\
\hline
\end{tabular}

P: P value S: Significativo NS: No significativo.

cópica ha ganado interés de los pacientes y médicos como alternativa quirúrgica mínimamente invasiva.

En nuestro estudio destacan las diferencias significativas encontradas en términos de tiempo operatorio, a favor de la cirugía convencional, y del índice de transfusiones y tiempo de sonda Foley a favor del abordaje laparoscópico. En el análisis del tiempo operatorio, creemos que esta variable está directamente relacionada a la curva de aprendizaje del 
Tabla 3. Complicaciones presentadas en prostatectomía radical laparoscópica y retropúbica en el Servicio de Urología del Hospital DIPRECA, (en negritas las derivadas del manejo de la sonda foley en el post operatorio)

\begin{tabular}{lccc}
\hline Complicaciones & $\begin{array}{c}\text { Prostatectomía } \\
\text { retropúbica }\end{array}$ & $\begin{array}{c}\text { Prostatectomía } \\
\text { Laparoscópica }\end{array}$ & $\begin{array}{c}\text { Valor } \\
\mathbf{d e ~ p}\end{array}$ \\
\hline Retención Urinaria & 1 & 5 & 0,1051 \\
Filtración Urinaria & - & 2 & 0,2309 \\
Hemorragia & 1 & 3 & 0,3517 \\
Perforación recto & - & 1 & 0,4828 \\
Estenosis cuello & 5 & 1 & 0,2081 \\
Seroma & 1 & - & 1,0 \\
TVP & - & 1 & 0,4828 \\
Hipercapnia intra operatoria & - & 1 & 0,4828 \\
Ave Embólico & - & 1 & 0,4828 \\
Técnica QX & 7 & 10 & 0,4339 \\
Sonda Foley & 1 & 5 & 0,1051 \\
Total & 8 & 15 & 0,1019 \\
\hline
\end{tabular}

cirujano; en este sentido, nuestra serie, al igual que las existentes en la literatura, compara una técnica establecida y conocida por la mayoría de los urólogos (PRR) versus el abordaje laparoscópico, que continúa en desarrollo y que en nuestro estudio representa la experiencia inicial de uno de los autores.

El menor sangrado intra operatorio es significativamente menor en la PRL, esto se debe a que la presión de insuflación de dióxido de carbono, usada para realizar la cavidad, produce el colapso de venas de pequeño calibre disminuyendo considerablemente el sangrado en napa. Otra variable es que al utilizar una visión de aumento que puede llegar hasta los 10x, la hemostasia es más prolija que en la cirugía convencional. El menor tiempo de sonda Foley a favor de la PRL se debe a que la anastomosis uretro vesical se realiza completamente bajo visión directa, pudiendo colocar tantos puntos como fuese necesario hasta dejar una unión impermeable. En la PRR esto no es posible ya que al bajar la vejiga hacia la posición de anastomosis, la uretra queda fuera de la visión del cirujano, por tanto la anastomosis se realiza primero colocando los puntos sin anudar en base a un esquema que en nuestro servicio es de 5 puntos, y luego se desciende la vejiga hasta su posición definitiva y se anudan los puntos a ciegas. Esto hace que el cirujano no tenga la certeza de que la anastomosis haya quedado impermeable y que se asegure más tiempo de sonda Foley en el post operatorio.
Si bien el resto de las variables no son estadísticamente significativas, observamos que en la PRL el tiempo de hospitalización es menor y se debe a la rápida recuperación que experimentan los pacientes, con escasos requerimientos de analgesia y deambulación precoz. En cuanto al análisis de las complicaciones, estas fueron separadas en las relacionadas a la técnica quirúrgica y a las derivadas del retiro de la sonda Foley. Hicimos esta separación, ya que durante el desarrollo de la técnica de la PRL existieron cambios de conducta respecto al retiro de la sonda Foley. En las primeras series mundiales se recomendaba un retiro precoz basado en que la anastomosis quedaba probadamente impermeable en el intra-operatorio y por tanto no era necesario proteger dicha unión por tantos días, así podemos citar trabajos como el de Abbou et $\mathrm{al}^{6}$ que recomendaba el retiro precoz de la sonda a los 2 días post operatorio. La experiencia fue demostrando que en algunos pacientes se producía una retención urinaria por edema de la anastomosis con posterior filtración por globo vesical; esto fue llevando a prolongar el retiro de la sonda Foley hasta llegar a la actualidad donde existe consenso mundial de que debe hacerse entre el $7^{\circ}$ y $10^{\circ}$ día.

En nuestra serie, la mayor frecuencia de complicaciones en PRL se asocia al desarrollo de la curva de aprendizaje de la técnica quirúrgica y creemos que aún cuando no son significativas, éstas deberían igualarse a las de la PRR en la medida que la experiencia vaya en aumento.

Los resultados funcionales postoperatorios de la prostatectomía radical como continencia urinaria y función eréctil junto con un adecuado control oncológico de la enfermedad, son herramientas y pilares fundamentales del resultado quirúrgico, sea éste por vía retropúbica o laparoscópica. En nuestro estudio no hubo diferencias significativas entre las 2 técnicas en el análisis de los resultados oncológicos como los funcionales, si bien hay tendencias a favor de una $\mathrm{u}$ otra técnica, todavía no hay un claro predominio de alguna en lo referente a potencia, continencia, márgenes quirúrgicos positivos y recurrencia al igual que otras series comparativas publicadas ${ }^{7-14}$. 
Al igual que en este estudio, los trabajos similares existentes en la literatura poseen el sesgo de comparar una técnica en desarrollo como la PRL versus una técnica estandarizada y común en la práctica urológica como la PRR. En este sentido las publicaciones realizadas por Rasweiller et al 15-16 muestran una clara tendencia de paso de conocimientos de generación en generación de urólogos laparoscopistas con una clara disminución de la curva de aprendizaje, que se traduce en menor tiempo operatorio y menor porcentaje de complicaciones. En Chile los urólogos de la $2^{\mathrm{a}}$ generación se encuentran finalizando su curva de aprendizaje.

\section{CONCLUSIONES}

Creemos que la prostatectomía radical retropúbica es una técnica que llegó a su punto máximo de rendimiento. La prostatectomía radical laparoscópica está en sus comienzos, en nuestro país recién la segunda generación de urólogos está terminando su curva de aprendizaje. Comparando ambas técnicas en condiciones semejantes, la prostatectomía retropúbica sólo aventaja a la prostatectomía radical laparoscópica en tiempo operatorio, estando esta variable directamente relacionadas a la curva de aprendizaje. Ambas técnicas son comparables y factibles de realizar y creemos que con el tiempo, se presentará una tendencia, que aventajara la prostatectomía radical laparoscópica sobre la retropúbica.

Según nuestra revisión, este es el primer trabajo comparativo entre estas dos técnicas que se publica en Chile.

\section{REFERENCIAS}

1. Guazzoni G, Cestari A, Naspro R, Riva M, Centemero A, Zanoni M, Rigatti L, Rigatti P.Intra- and Peri-Operative Outcomes Comparing Radical Retropubic and Laparoscopic Radical Prostatectomy: Results from a Prospective, Randomised, SingleSurgeon Study. Eur Urol. 2006 Jul;50(1):98-104.

2. Rassweiler J. Open vs. Laparoscopic Radical Prostatectomy and Laparoscopy is Better. Eur Urol. 2006 Jul;50(1):26-28.

3. Wagner AA, Link RE, Trock BJ, Sullivan W, Pavlovich CP. Trock, Wendy Sullivan, And Christian P. Pavlovich. Comparison of Open and Laparoscopic Radical Prostatectomy Outcomes from a Surgeon's Early Experience. Urology 2007;70(4): 667-671.

4. Schuessler WW, Schulam PG, Clayman RV, Kavoussi LR. Laparoscopic radical prostatectomy: initial short-term experience. Urology 1997;50(6):854-857.
5. Guillonneau B, Vallancien G. Laparoscopic radical prostatectomy: initial experience and preliminary assessment after 65 operations. Prostate. 1999;39(1):71-75

6. Nadu A, Salomon L, Hoznek A, Olsson Le, Saint F, De La Taille A, Cicco A, Chopin D, Abbou CC. Early removal of the catheter after laparoscopic radical prostatectomy. Journal Urology 2001;166(5): 1662-1664.

7. Dahl DM, He W, Lazarus R, McDougal WS, Wu CL. Pathologic outcome of laparoscopic and open radical prostatectomy. Urology 2006; 68(6):1253-1256.

8. Park S, Jaffer O, Lotan Y, Saboorian H, Roehrborn CG, Cadeddu JA. Contemporary Laparoscopic and Open Radical Retropubic Prostatectomy: Pathologic Outcomes and Kattan Postoperative Nomograms Are Equivalent. Urology 2007; 69(1): 118-122.

9. Touijer K, Kuroiwa K, Eastham JA, Vickers A, Reuter VE, Scardino PT, Guillonneau B. Risk-Adjusted Analysis of Positive Surgical Margins Following Laparoscopic and Retropubic Radical Prostatectomy. European Urology. 2007;52(4):10901096.

10. G. Janetschek AF. Montorsi. Open versus Laparoscopic Radical Prostatectomy. European Urology supplements 2006; 5: 377384.

11. Aristotelis G. Anastasiadis, Laurent Salomon, Ran Katz, Andras Hoznek, Dominique Chopin, And Clement-Claude Abbou. Radical retropubic versus laparoscopic prostatectomy: a prospective comparasion of functional outcome. Urology 2003;62: 292-297.

12. Remzi M, Klingler HC, Tinzl MV, Fong YK, Lodde M, Kiss B et al. Morbidity of Laparoscopic Extraperitoneal versus Transperitoneal Radical Prostatectomy versus Open Retropubic Radical Prostatectomy. European Urology 2005; 48:83-89.

13. Laurent Salomon, Olivier Levrel, Alexandre De La Taille, Aristotelis G, et al. Radical prostatectomy by the retropubic, perineal, and laparoscopic approach: 12 years of experience in one center. European Urology 2002; 42: 104-111.

14. Joel B. Nelson. Debate: Open radical prostatectomy vs. laparoscopic vs. robotic. Urologic Oncology: Seminars and Original Investigations 2007;25:490-493.

15. Frede T, Erdogru T, Zukosky D, Gulkesen H, Teber D, Rassweiler J. Comparison of training modalities for performing laparoscopic radical prostatectomy: experience with 1,000 patients. Journal Urology. 2005;174(2):673-678.

16. Rassweiler J, Klein J, Teber D, Schulze M, Frede T. Mechanical simulators for training for laparoscopic surgery in urology. Journal Endourology. 2007;21(3): 252-262.

Correspondencia autor: Dr. Marcelo Kerkebe Servicio de Urología. Hospital DIPRECA Avenida Vital Apoquindo 1200 - Las Condes - Chile Tel.: 056-2-2401412

E-mail autor: marcelo.kerkebe@gmail.com Información artículo: Original - Laparoscopia Trabajo recibido: noviembre 2008

Trabajo aceptado: diciembre 2008 